\title{
THE MATHEMATICAL THEORY OF A CLASS OF SURFACE WAVE ANTENNAS*
}

\author{
BY \\ JULIUS KANE \\ University of Rhode Island
}

In this paper we investigate a three part boundary value problem which is used as a mathematical model of a class of surface wave radiators. A feature of the analysis is that various items of physical interest, such as gain and radiation resistance, can be displayed as elementary expressions which are in accord with experimental data.

1. Introduction. If a time factor $e^{-i \omega t}$ is suppressed, then Maxwell's equations in free space assume the form

$$
\operatorname{curl} \mathbf{E}=+i \omega \mu_{0} \mathbf{H}, \quad \operatorname{curl} \mathbf{H}=-i \omega \epsilon_{0} \mathbf{E} .
$$

These equations admit the existence of electro agnetic fields known as surface waves. These waves are so-called because they prop $\_$ate tangentially to specially-treated surfaces and decay along any outward normal to that surface. In two dimensions, if $H_{x}=H_{\nu}=0$, and $H_{z}=u(x, y)$, the $T M$ polarization, then the vector equations (1) are equivalent to the reduced scalar wave equation

$$
\left(\nabla^{2}+k^{2}\right) u(x, y)=0, \quad k^{2}=\omega^{2} \epsilon_{0} \mu_{0},
$$

and the statements

$$
E_{x}=\frac{-1}{i \omega \epsilon_{0}} \frac{\partial u}{\partial y}, \quad E_{y}=\frac{+1}{i \omega \epsilon_{0}} \frac{\partial u}{\partial x}, \quad E_{z}=0 .
$$

The wave equation (2) admits the field

$$
u=\exp \left[i\left(k^{2}+\lambda^{2}\right)^{1 / 2} x-\lambda y\right]
$$

where $\lambda$ is an arbitrary parameter, as an acceptable solution: if $\lambda$ has a negligible imaginary part, then this wave function is the simplest example of a surface wave. In this case, it propagates in the positive $x$-direction, and is exponentially attenuated in the upper half space $y \geq 0$. In engineering contexts, $\lambda$ is related to the impedance $z=R-i X$ of the surface, here the $x, z$-plane, by the relation

$$
\lambda=i k Z=i k(R-i X) .
$$

It is not difficult to prepare a reactive surface, that is, one for which $R$ is negligible in comparison to $X$. For these structures, $\lambda \approx k X$, and can be considered as a positive real constant; this restriction will be assumed in the subsequent analysis. The complex nature of the supporting surface, the $x, z$-plane, can then be mathematically abstracted as an impedance relation, or as a mixed boundary condition of the form

$$
\frac{\partial u}{\partial y}+k X u=0,
$$

as has been done by many authors [1] through [5].

${ }^{*}$ Received Jan. 11, 1963. The research described in this report has been sponsored by the Electronics Research Directorate of the Air Force Cambridge Research Laboratories, Division of Aerospace Research, under contract number AF 19(604)-7983. 
It is clear by an inspection of (4), that the larger $\lambda=k X$ is, the more tightly bound is the surface wave (4) to the $x, z$-plane. Furthermore, it has been shown by Cullen [6], Brick [7], Friedman [3], Kane [4], et. al., that the efficiency of surface wave excitation by a fixed source varies directly with increasing $X$. On the other hand, truncated reactive surfaces are useful in antenna applications: The treated surface serves as a transmission line which delivers the surface wave to the termination where part of the energy of the surface wave is radiated. A figure of merit in this application is gain, and it has been theoretically shown by Kay [2] that this parameter is $\mathbf{G}_{\infty}=4 / X$ for a surface wave incident from infinity upon a termination consisting of a conducting ground plane. Since practical surface wave antennas include excitation, transmission, and radiation regions, their design is restricted by these conflicting demands upon $X$. Furthermore, limited theoretical knowledge has been afforded the antenna engineer since realistic mathematical models pose intractable boundary value problems. In this report we obtain an explicit, if approximate, solution of a three-part boundary value problem motivated by questions of antenna design. With this information we are then able to discuss the gain of a hypothetical antenna which includes surface-wave excitation and radiation regions. We find, for example, that although this gain still varies inversely with the reactance, it approaches a finite limit as the reactance $X$ vanishes.

2. Formulation. We seek a solution of the reduced wave equation (see Fig. 1)

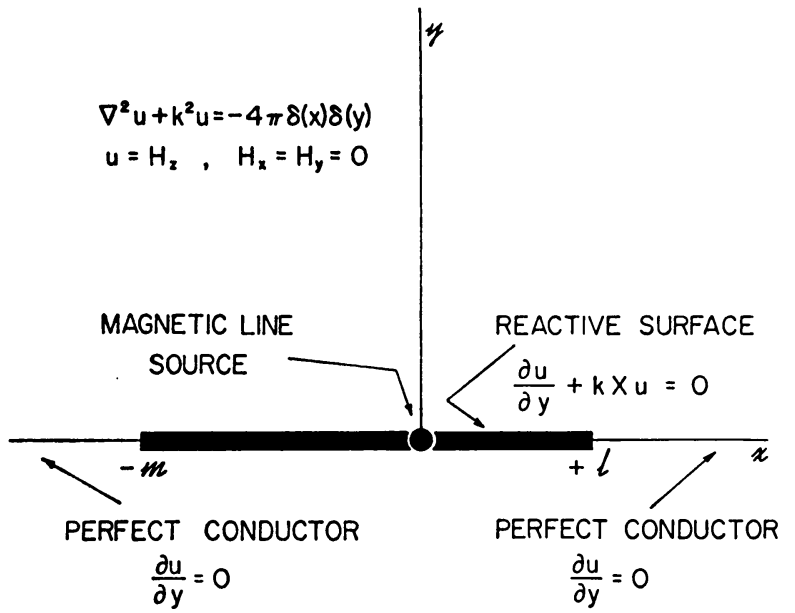

Fig. 1. The geometry of our problem: A reactive surface which extends from $x=-m$ to $x=l$ is excited at the origin by a magnetic line dipole source. If we restrict our region of interest to the upper half space, then the boundary conditions on the $x$-axis for $x>l, x<-m$ are appropriate for perfect conductors. Alternatively, by analytic continuation of $u$, these conditions can be thought of as implying that the $x$-axis is a plane of symmetry in the whole space. (The boundary condition on the lower portion of the reactive section would be $\partial u / \partial y-k X u=0$ owing to the change in sign in the normal derivative after reflection). Furthermore, in the symmetric problem $l=m$, the positive $y$-axis can be thought of as a metallic sheet since $\partial u / \partial x=0$ along that ray; this situation would correspond to a surface wave antenna placed in a corner reflector.

$$
\left(\nabla^{2}+k^{2}\right) u(x, y)=-4 \pi \delta(x) \delta(y), \quad y \geq 0
$$

which satisfies the three-part boundary conditions 


$$
\begin{array}{lrr}
\frac{\partial u}{\partial y}=0 & y=0, & x>l, \\
\frac{\partial u}{\partial y}+k X u=0 & y=0, & -m<x<l, \\
\frac{\partial u}{\partial y}=0 & y=0, & x>l,
\end{array}
$$

and behaves like an outgoing wave at infinity. If $l=m$, then the problem posed by equations (6) and (7) is suggestive of the cross section of a cylindrical beacon antenna of a type considered by Plummer [8]: A line source at the vertex excites surface waves on the reactive portion modeled by the boundary condition (7a); the treated surface terminates at $x=-m$, and $x=l$ into perfect conductors $\left(u_{y}=0\right)$ which extend to. $x=-\infty$, and $x=+\infty$ respectively. Alternatively, the boundary conditions on the metallic portions can be interpreted as implying symmetry about the $x, z$-plane.

Before constructing a formal mathematical solution it is convenient to consider the operation of the antenna in the time-domain, and suppose the source has just been turned on. At first the field that surrounds the source would be the same as that on an infinite reactive plane since the field would not have reached either termination. This timevarying field would be a Fourier transform of a known [1] time-harmonic field which consists of two parts: a cylindrical radiated field

$$
C_{0}=i \pi\left[H_{0}^{(1)}(k r)-k X e^{-k X y} \int_{-\infty}^{y} e^{k X t} H_{0}^{(1)}\left(k\left[x^{2}+t^{2}\right]^{1 / 2}\right) d t\right],
$$

and a surface wave field

$$
S W=\frac{2 \pi i X}{M} \exp (i k M|x|-k X y), \quad M=\left(1+X^{2}\right)^{1 / 2}
$$

It is especially worth noting that the pattern function $p(\theta)$ of any cylindrical field

$$
p(\theta) \frac{e^{i k r}}{(k r)^{1 / 2}}+O\left[(k r)^{-3 / 2}\right]
$$

must have a null in the direction of the reactive surface* (the Karp-Karal lemma). For our geometry this implies that the pattern function $p_{0}(\theta)$ of the cylindrical field (8) must satisfy

$$
p_{0}(0)=p_{0}(\pi)=0,
$$

so that the field which arrives at say the right termination $x=l$ is proportional to

$$
\exp (i M k l-k X y)+O\left[(k l)^{-3 / 2}\right]
$$

if $k l \gg 1$. That is, since surface wave antennas are at least several wavelengths long, the field that arrives at the termination is dominated by the surface wave field. If we can neglect terms of order $O\left[(k l)^{-3 / 2}\right]$ at the discontinuity, then the situation in these regions has been discussed in [2]. The diffraction pattern is known: a reflected surface wave is

\footnotetext{
*Essentially, the Karp-Karal lemma is an observation that any wave function $u$ whose leading term is asymptotically $g(\theta) /(k r)^{1 / 2}$ must have $g(0)=0$ if $u$ is to satisfy a boundary condition of the form $\partial u / \partial \theta+\lambda r u=0$ along the ray $\theta=0$.
} 
generated and a new cylindrical field appears at the termination. By the Karp-Karal lemma, this scattered cylindrical field will have a null in the direction of the reactive surface and $a$ fortiori the source. Clearly the situation is the same at the other terminaion, and, consequently, the fields that are returned to the source are the reflected surface waves plus portions of the cylindrical field which behave like terms of order $O\left[(k l)^{-3 / 2}\right]$. These reflected surface waves then meet a new discontinuity at the source and again further diffracted fields are excited. In the limit, there are multiply reflected surface waves between the source and terminations, plus cylindrical fields with phase centers at the origin and either termination. By the Karp-Karal lemma these cylindrical fields have pattern functions which must vanish on the reactive surface. Hence, apart from terms of order $O\left[(k l)^{-3 / 2}\right]$, the interaction between the source field and terminal fields arises from the multiply reflected surface waves. One principal result is to show that this coupling can be calculated in an elementary and explicit manner.

3. Solution. We shall obtain our principal result as a linear combination of the solutions to simpler problems, and determining the required coefficients. For this purpose, it is convenient to work backwards from the discontinuity: At the right-hand termination we choose the field to be of the form

$$
J_{l}\left\{\exp [i k M(x-l)-k X y]+R(X) \exp [-i k M(x-l)-k X y]+C_{l}\left(r_{l}, \theta_{l} ; X\right)\right\} \text {, }
$$

where $M=\left(1+X^{2}\right)^{1 / 2}, R(X)=\rho e^{i \phi}$ is the reflection coefficient for a unit surface wave incident upon a conducting ground plane, and $C_{l}\left(r_{l}, \theta_{l} ; X\right)$ is the diffracted cylindrical field excited by the surface wave, which has its phase center at the discontinuity in reactance $x=l, y=0$. That is, we include within (12), an incident and reflected surface wave, plus a cylindrical radiated field with phase center at the discontinuity $(l, 0)$. An analysis of these fields which exhibits the behavior of $R(X)$ and $C_{l}\left(r_{l}, \theta_{l} ; X\right)$ has been given by Kay [2], (see also Appendices A, B). Since the wave equation (6) is homogeneous in a vicinity of the termination at $(l, 0)$, the wave function (12) in square brackets can be multiplied by an as yet unknown constant $J_{l}$, and remain a wave function. Likewise, at the left-hand termination we introduce

$$
\begin{aligned}
& J_{-m}\{\exp [-i k M(x+m)-k X y] \\
& \left.\quad+R(X) \exp [i k M(x+m)-k X y]+C_{-m}\left(r_{-m}, \theta_{-m} ; X\right)\right\},
\end{aligned}
$$

which includes another unknown factor $J_{-m}$. In the vicinity of the source, we superpose the cylindrical portion of Karp and Karal's solution [1]

$$
C_{0}(r, \theta, X)=i \pi\left[H_{0}^{(1)}(k r)-k X e^{-k X y} \int_{-\infty}^{\nu} e^{k X t} H_{0}^{(1)}\left(k\left[x^{2}+y^{2}\right]^{1 / 2}\right) d t\right]
$$

of the inhomogeneous wave equation, and the solutions of the homogeneous wave equation (12) and (13). The field (14) has the required singularity at the origin, and is a wave function everywhere in the upper-half plane, except along the line $x=0$, where it is continuous, but has a jump $J_{0}$ in the normal derivative of magnitude

$$
J_{0}=\left.\frac{\partial C_{0}}{\partial x}\right|_{0-} ^{0+}=-4 \pi k X e^{-k X y} .
$$

Note that the $y$-variation of the jump in (15) is proportional to the $y$-variation of a surface wave. We exploit this fact by using the surface wave portions of (12) and (13) as "patch functions." That is, let the surface wave portions of (12) and (13) exist only for 
$0 \leq x \leq l$, and $-m \leq x \leq 0$ respectively. Then the total field described by (12), (13) and (14) will be discontinuous across the line $x=0$, and will have a discontinuity in the normal derivative there, unless $J_{l}$ and $J_{-m}$ are selected properly. Clearly $J_{l}$ and $J_{-m}$ must be so chosen that the combination (12), (13) and (14) is continuous, and possesses continuous first derivatives in any neighborhood of the $y$-axis. For continuity we need choose

$$
J_{l}\left(e^{-i k M l}+\mathrm{Re}^{i k M l}\right)-J_{-m}\left(e^{-i k M m}+\mathrm{Re}^{i k M m}\right)=0,
$$

and for a continuous normal derivative we must have

$$
M\left[J_{l}\left(e^{-i k M l}-\operatorname{Re}^{i k M l}\right)-J_{-m}\left(-e^{-i k M m}+\operatorname{Re}^{i k M m}\right)\right]=4 \pi X .
$$

We can solve (16) and (17) for $J_{\imath}$ and $J_{-m}$

$$
\begin{aligned}
J_{l} & =\frac{4 \pi i X\left(e^{-i k M m}+\mathrm{Re}^{i k M m}\right)}{M \cdot \operatorname{det}}, \\
J_{-m} & =\frac{4 \pi i X\left(e^{-i k M l}+\mathrm{Re}^{i k M l}\right)}{M \cdot \operatorname{det}},
\end{aligned}
$$

where

$$
\operatorname{det}=\left|\begin{array}{rr}
\left(e^{-i k M l}+\operatorname{Re}^{i k M l}\right) & -\left(e^{-i k M m}+\operatorname{Re}^{i k M m}\right) \\
\left(e^{-i k M l}-\operatorname{Re}^{i k M l}\right) & \left(e^{-i k M m}-\operatorname{Re}^{i k M m}\right)
\end{array}\right|,
$$

and then we can display our solution as

$$
\begin{aligned}
u(x, y) & =C_{0}(r, \theta ; X)+J_{l} C_{l}\left(r_{l}, \theta_{l} ; X\right)+J_{-m} C_{-m}\left(r_{-m}, \theta_{-m} ; X\right) \\
& +J_{l}\{\exp [i k M(x-l)]+R \exp [-i k M(x-l)]\} e^{-k X_{y}} U(x) U(x-l) \\
& +J_{-m}\{\exp [-i k M(x+m)]+R \exp [i k(x+m)]\} e^{-k X_{y}} U(-x) U(x+m),
\end{aligned}
$$

where $U(x)$ is the Heaviside unit function

$$
U(x)=\left\{\begin{array}{l}
0 \text { for } x<0 \\
1 \text { for } x>0
\end{array}\right.
$$

4. Discussion of the solution. A. Introduction. To simpify the subsequent analysis it is very convenient to discuss a symmetrical antenna, i.e., one for which $l=m$. With this choice

$$
J=J_{\iota}=J_{-\imath}=\frac{2 \pi i X \exp (i M k l)}{M[1-R(X) \exp (2 i M k l)]},
$$

and the field on the antenna is

$$
\begin{aligned}
u(x, 0)=i \pi & {\left[H_{0}^{(1)}(k x)-k X \int_{-\infty}^{0} e^{k X t} H_{0}^{(1)}\left(k\left[x^{2}+t^{2}\right]^{1 / 2}\right) d t\right] } \\
& +J\left[C_{l}(l-x, \pi ; X)+C_{-l}(l+x, 0 ; X)\right] \\
& +J \exp (-i M k l)[\exp (i k M|x|)+R(X) \exp (2 i M k l-i k M|x|)] .
\end{aligned}
$$

for $y=0,|x| \leq l$. Although the literature [2] is explicit on such questions as the magnitude $R(X)$ of the reflection coefficient and the magnitude $p_{\Delta_{l}}(\theta)$ of the pattern functions 
of the terminal radiation fields, there is no elementary expression which gives the phase of these important quantities. This situation arises from the fact that these parameters emerge as part of involved function-theoretic expressions required to solve a WienerHopf problem. Kay has been successful in showing that the magnitude of the required parameters can be presented in an elementary form, but no similar result is available for the phase. In Appendix B we show how the required factorization can be performed approximately, with a small error for $|X|<1$. The feature of this procedure is that it yields an explicit elementary representation for the phase whose accuracy is as high as the theory justifies. In particular, we find that the reflection coefficient can be written as

$$
R(X)=\rho e^{-i \phi}
$$

wherein

$$
\rho=\frac{X^{2}}{1+\left(1+X^{2}\right)^{1 / 2}+X^{2}}, \quad \phi=2 \tan ^{-1}\left\{\frac{\sinh ^{-1} X}{\pi}\right\} .
$$

Since the exact magnitude $\rho$ is known, we introduce that expression in (24), and use the approximate result only in the phase $\phi$.

B. Maximizing the end-fire field. For an antenna which is symmetrical about the $y$-axis, maximizing the field in one end-fire direction achieves a similar result in the other end-fire direction. Let us consider the radiation field towards the right: Both the source pattern function $p_{0}(0)$ and the left-hand pattern function $p_{-l}(0)$ have nulls in this direction, and hence the only contribution to the combined pattern function $P(\theta)$ for $\theta=0$ arises from the right hand pattern function $p_{l}(0)$. This quantity varies directly as the amplitude of the incident surface wave which is given by (21). The magnitude of this expression will be a maximum when the electrical length $M k l$ of the antenna is such that

$$
1-\rho e^{-i \phi} e^{2 i M k l}=1-\rho
$$

or, by (24),

$$
-\phi+2 M k l=-2 \tan ^{-1}\left\{\frac{\sinh ^{-1} X}{\pi}\right\}+2 M k l=n(2 \pi), \quad n=0,1,2, \cdots .
$$

That is, for maximum field in the end-fire direction we should choose the electrical length $M k l$ of the antenna to be

$$
(M k l)_{\max }=n \pi+\tan ^{-1}\left\{\frac{\sinh ^{-1} X}{\pi}\right\}
$$

Analogously, the minimum field arises when

$$
(M k l)_{\min }=\frac{n \pi}{2}+\tan ^{-1}\left\{\frac{\sinh ^{-1} X}{\pi}\right\}
$$

Most surface wave antennas operate with small reactances; for these cases

$$
(M k l)_{\max } \approx n \pi, \quad X \ll 1,
$$

or the phase separation between the termination and source behaves like some multiple of $\pi$ for maximum gain.

Consider first the odd multiples of $\pi$ in equation (29). For antennas which are so 
adjusted, the end-fire field will be a maximum. However, just off the end-fire direction, the source and other terminal field are no longer null, and hence interfere with the main lobe. As a result, the combined half-power beam width of such an antenna will be less than that of an isolated surface wave radiating at a discontinuity in reactance. This point is in agreement with the rather general experimental observation that amplitude patterns for antennas are about 20 per cent narrower than Kay's theoretical radiation pattern for an ideal surface wave, incident from infinity, radiating at a discontinuity [10]. In Figure 2 we plot the phase separation between source and termination which maximizes the end-fire amplitude according to (27) for odd $n$.

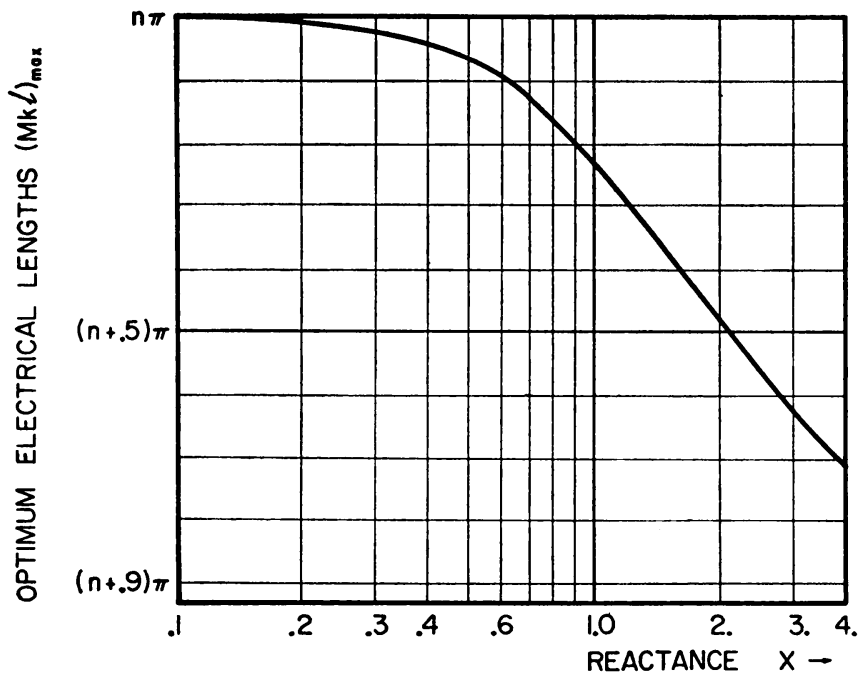

FIG. 2. The optimum electrical lengths required to maximize the amplitude of the outward surface wave as given by equation (27).

On the other hand, even multiples of $\pi$ are also acceptable for (29). These values of $M k l$ would yield the same end-fire amplitude, but the combined pattern would be broader than the Kay pattern, since the fields now add. This adjustment is of less interest for broader patterns are usually less desirable.

C. Definition of the gain. If a semi-infinite reactive surface is terminated by a perfectly conducting half plane, then the end fire gain including mismatch losses of the radiation field produced by an incident surface wave can be shown [2] to be

$$
\mathrm{G}_{\infty}=4 / X
$$

where $X$ is the reactance of the surface. The expression (30) is a consequence of the following definition of gain

$$
\mathbf{G}_{\infty}=\frac{\text { power in the end-fire direction at a distance } r \text { from the discontinuity }}{\text { total incident power } / \pi k r} .
$$

That is, $\mathbf{G}_{\infty}$ is taken to be the ratio of the end-fire power amplitude to the power amplitude that would arise if the total energy in the incident field were to be distributed 
isotropically in the upper half space. The expression (30) would apply to our problem only if all of the energy supplied by the radiating line source went into the surface wave field. Since in addition there are finite cylindrical fields, we can multiply (30) by an efficiency factor to get a definition of gain suitable for our purposes

$$
\mathbf{G}_{l} \equiv \frac{\text { power in outward } S W \text { field }}{\text { total radiated power }} \mathbf{G}_{\infty} \text {. }
$$

We use the power in the outward surface wave field in (32) since $\mathbf{G}_{\infty}$ includes mismatch losses.

D. Radiation Resistance. The formula (32) becomes quite tractable if we introduce the notion of radiation resistance: If $g$ is the strength of the line source, then the total radiated power would be $|\mathscr{I}|^{2} R$ where $R$ is the radiation resistance of the combination. Let $Q$ be the amplitude of the outward surface wave generated by a source of unit current, then a current $g$ will produce the outward surface wave

$$
H_{z}^{s w}=g Q \exp (i k M x-k X y)
$$

on the right arm of the antenna. By Maxwell's equation (3b) the Poynting flux $\Pi_{s w}$ carried by this field is

$$
\Pi_{s w}=\int_{0}^{\infty} E_{y}^{s w}\left(H_{z}^{s w}\right)^{*} d y=\frac{|g Q|^{2} M}{2 \omega \epsilon_{0} X},
$$

and then equation (32) becomes

$$
\mathrm{G}_{l}=\frac{2|a|^{2} M}{\omega \epsilon_{0} X \Re}
$$

which would be an explicit formula for the gain if the radiation resistance $R$ were known. Usually, complicated procedures are required to obtain the radiation resistance $R$. However, there is a very useful result originally due to Newstein and Lurye [10] and proved independently by Karp [11] in a more comprehensive form. For our purposes we shall state the result in form of the following lemma.

Lemma: Let the magnetic field $H_{z}$ in the region of a source at the origin have the form

$$
H_{z}=\left[H_{0}^{(1)}(k r)+\Psi(r, \theta)\right] e^{-i(\omega t+\delta)}, \quad H_{x}=H_{y}=0,
$$

where $\Psi(r, \theta)$ is regular in a neighborhood of $r=0$, and $\delta$ is some arbitrary constant phase factor. Then the power $\Pi$ radiated by the source is

$$
\Pi=\frac{2}{\omega \epsilon_{0}}[1+\Re e \Psi(0,0)] .
$$

In other words, if the phase factor $\delta$ has been so chosen that the field singularity is in the imaginary part of the spatial field, then the power radiated depends upon the value of the real part of the spatial field at the origin.

As an immediate consequence, since the radiation resistance $R$ is proportional to the radiated power, we have

$$
Q=\frac{2 C}{\omega \epsilon_{0}}[1+\operatorname{Re} \psi(0,0)],
$$


where $C$ is an unimportant dimensionality factor. We shall choose $C$ to be unity; this corresponds to a choice of units in which a magnetic line dipole source located on an infinite metallic ground plane has its gain normalized to unity. If we neglect terms of order $(k x)^{-3 / 2}$, then it is an elementary calculation to determine the finite part $\Psi(0,0)$ at the origin for our analysis. From equation (22) we obtain

$$
R=\frac{2}{\omega \epsilon_{0}}\left\{1-\frac{X}{M}+\frac{2 X}{M} \cdot R e\left[\frac{1+\rho \exp [i(\phi+2 M k l)]}{1-\rho \exp [i(\phi+2 M k l)]}\right]\right\} .
$$

The first two terms within the curly braces are the contributions of the free space and diffracted cylindrical fields to the radiation resistance. The last term is that portion of the resistance due to surface wave excitation on the antenna: It is very interesting to note that this term has the same form as the input resistance of a transmission line terminated by an impedance; the load in this case being the radiation and reflection of the surface wave at the termination. Observe that for fixed $X$, the only variation in the radiation resistance $R$ with changing length $l$ arises from this last term through a factor $e^{2 i M k}$, and that the radiation resistance $R$ goes through the following upper and lower bounds

$$
R_{\max }=\frac{2}{\omega \epsilon_{0}}\left(1-\frac{X}{M}+\frac{2 X}{M} \cdot \frac{1+\rho}{1-\rho}\right), \quad R_{\min }=\frac{2}{\omega \epsilon_{0}}\left(1-\frac{X}{M}+\frac{2 X}{M} \cdot \frac{1-\rho}{1+\rho}\right),
$$

as $l$ varies. These expressions can be compared with the resistance

$$
\Re_{\infty}=\frac{2}{\omega \epsilon_{0}}\left(1-\frac{X}{M}+\frac{2 X}{M}\right)
$$

which arises if the arms of the antenna are infinite in extent, or so terminated that the reflection coefficient $\rho$ is null; note that $R_{\min } \leq R_{\infty} \leq R_{\max }$.

$E$. Gain Variations. The remaining element required to specify the gain is the amplitude $Q$ of the outward surface wave. From (22) this parameter is seen to be

$$
a=\frac{J \exp (-i M k l)}{\pi i}=\frac{2 X}{M\{1-\rho \exp [i(\phi+2 i M k l)]\}} .
$$

This term $Q$, like $R$, varies in magnitude through a factor $e^{2 i M k l}$ as a function of $l$. Comparing (39) and (42) we find that the extremum values $\mathbb{R}_{\substack{\max \\ \text { min }}}$ and $\mathfrak{Q}_{\substack{\max \\ \text { min }}}$ arise simultaneously for the same values of $l$.

Since the gain $\mathbf{G}_{l}$ depends upon $|a|^{2} / \mathcal{R}$ it can easily be shown that the maxima and minima of $\mathbf{G}_{l}$ too arise for these values of $l$. As a result, because $\left.\left|a_{\max }\right|^{2} / \mathcal{R}_{\max }\right\rangle$ $\left|a_{\min }\right|^{2} / Q_{\min }$ we obtain

$$
\begin{aligned}
& \left(\mathbf{G}_{l}\right)_{\max }=\frac{\cdot}{(1-\rho)^{2}[M-X+2 X(1+\rho) /(1-\rho)]}, \\
& \left(\mathbf{G}_{l}\right)_{\min }=\frac{4}{(1+\rho)^{2}[M-X+2 X(1-\rho) /(1+\rho)]}
\end{aligned}
$$

where $\rho$ is given by (24). For small $X$ it is clear that

$$
\left(\mathbf{G}_{l}\right)_{\max } \approx\left(\mathbf{G}_{l}\right)_{\min } \approx 4 \quad X \ll 1
$$

and for large $X$ the reactive surface is ineffective in increasing the gain since 


$$
\left(\mathrm{G}_{l}\right)_{\max } \approx\left(\mathrm{G}_{l}\right)_{\min } \approx \frac{4}{2 X\left(1-\rho^{2}\right)}+1, \quad X \gg 1 .
$$

These last two relations also indicate that for extreme values of $X$, there is little variation between $\left(\mathbf{G}_{l}\right)_{\max }$ and $\left(\mathbf{G}_{l}\right)_{\min }$; for small $X$ this situation is a consequence of the reflection coefficient $\rho$ being small, whereas for large $X$, the anticipated large excursions of gain [based upon equation (42)] are damped by the wide variations in the radiation resistance R. In Fig. 3 we plot the possible limits of $\mathrm{G}_{l}$ as a function of $X$. We observe

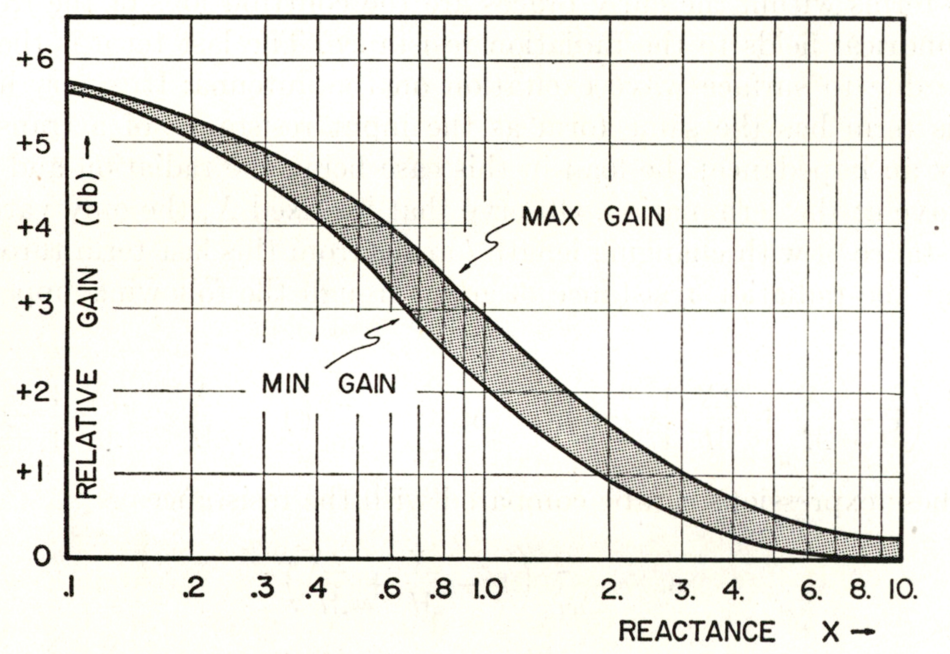

FIG. 3. The gain variations $10 \log \left(\mathbf{G}_{l}\right)_{\max }$ according to equation (43) plotted in decibels. $\min$

that there is no relative maximum for $\left(\mathbf{G}_{l}\right)_{\max }$, and that it decreases monotonically for increasing $X$. Furthermore, the gain is finite for $X=0$, unlike Kay's gain $\mathbf{G}_{\infty}$. However, this result should not be interpreted as implying that surface wave antennas are gainlimited: we have obtained a gain of 4 (or $6 \mathrm{db}$ ) as a limiting value only because the surface wave excitation efficiency of our omnidirectional line source falls off as $X$ with decreasing reactance. There is no reason why other directive feeds could not be more efficient as surface wave exciters [4], and produce gains as high as desired (however, there is another limitation which will be discussed in the next section).

In Fig. 4 we plot the variations in the normalized radiation resistance $R$ as a function of $X$. It is these excursions in $R$ which keep the gain variations small. This explains why the surface wave antenna is not a resonant device. Although maximum gain does depend upon the electrical length $M k l$, we have seen that this adjustment is not critical, indeed from Fig. 3 the maximum variation in gain is seen to be less than $1 \mathrm{db}$; this agrees with the experimental conclusion that the observed gain variations are also less than $1 \mathrm{db}$. [9, p. 16-13].

$F$. Surface wave purity. Our analysis in the preceding sections has implied that the gain of a surface wave antenna depends only upon its electrical length $M k l,(\bmod 2 \pi)$ and not its physical length $l$. In actual practice, it is known that if a surface wave antenna is to operate, then it must exceed some minimum length. This minimum length is that distance from the source at which the surface wave has peeled itself off from the 


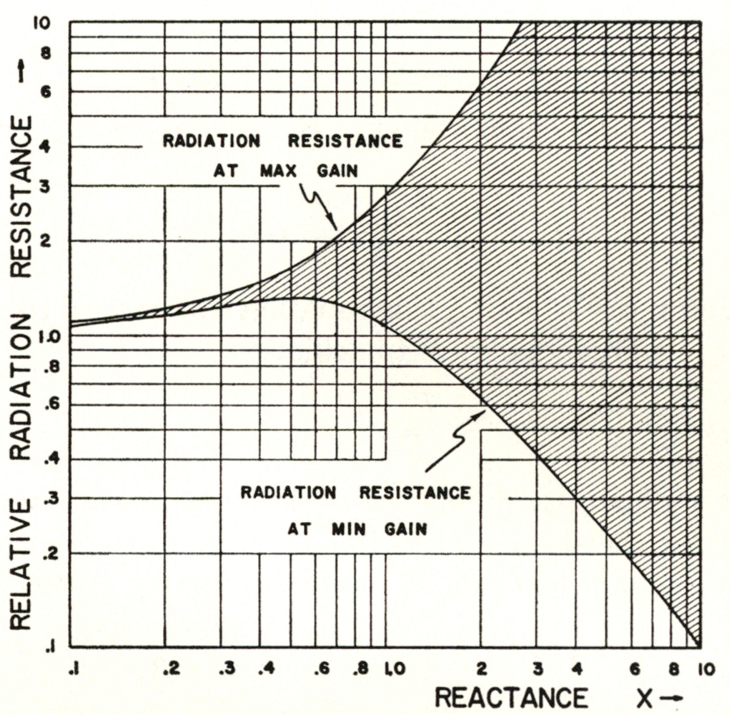

FIG. 4. The normalized radiation resistance as given by equation (40).

cylindrical wave. We have tacitly assumed that we could ignore the cylindrical field on the antenna's surface by the Karp-Karal lemma. However, terms of order $O\left[(k x)^{-3 / 2}\right]$ can not be neglected at moderate distances from the source. It is worthwhile to calculate that distance from the excitation at which the surface wave predominates. For this purpose let us ignore the effects of the termination and consider the purity of the surface wave field as a function of distance along the reactive surface measured from the magnetic line dipole source at the origin. A purity factor $P(x, X)$ can be defined as

$$
P(x, X)=\left[\left|H_{z}^{S W}\right| /\left|H_{z}^{c y l}\right|\right]_{y=0}
$$

which is a function of the distance $x$ from the source, and the reactance $X$ of the surface. If the reactive surface is unbounded, then from Eq. (22), with $R=0$, and $l=\infty$ we obtain

$$
\left.H_{z}^{c y l}\right|_{y=0}=\pi i\left[H_{0}^{(1)}(k x)-I\right],
$$

where

$$
\begin{gathered}
I=k X \int_{-\infty}^{0} e^{k X} H_{0}^{(1)}\left(k\left[x^{2}+\zeta^{2}\right]^{1 / 2}\right) d \zeta, \\
\left.H_{z}^{S W}\right|_{y=0}=\frac{2 \pi i X}{\left(1+X^{2}\right)^{1 / 2}} e^{i M|k x|},
\end{gathered}
$$

so that

$$
P=\frac{2 X}{\left(1+X^{2}\right)^{1 / 2}\left|H_{0}^{(1)}(k x)-I\right|}
$$

In Appendix $\mathrm{C}$ we obtain an estimate for $I$, if $X \neq 0$, of the form

$$
I \approx H_{0}^{(1)}(k x)\left[1+2 i /\left(X^{2} k x\right)\right]
$$

The leading term of $I$ cancels the source field on the $x$-axis (as it should by the KarpKaral lemma), and thus 


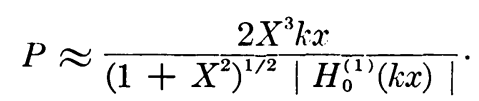

If we exclude the immediate neighborhood of the source we can replace $\left|H_{0}^{(1)}(k x)\right|$ by $(2 / \pi k x)^{1 / 2}$ whence

$$
P \approx\left(\frac{2 \pi}{1+X^{2}}\right)^{1 / 2} X^{3}(k x)^{3 / 2}
$$

For a given purity factor, say $P=P_{0}$, this equation can be used to estimate the required length of antenna $k x_{0}$, and reactance $X$ to achieve $P_{0}$

$$
k x_{0} \approx \frac{P_{0}^{2 / 3}}{X^{2}}\left(\frac{1+X^{2}}{2 \pi}\right)^{1 / 3}
$$

This formula clearly indicates why high gain-low reactance antennas must be longthey need the length for the surface wave to emerge from the source field.

Zucker has given an empirical rule of the thumb [9; eq. 16-19] for a surface wave to establish itself as

$$
k x_{0}^{Z}\left[\left(1+X^{2}\right)^{1 / 2}-1\right]=\pi / 3,
$$

which in essence states that $x_{0}^{z}$ is that distance from the feed at which the space wave, propagating at the velocity of light, leads the surface wave by $60^{\circ}$. If we expand the radical in (53) that expression takes the form

$$
k x_{0}^{z} \approx(2 \pi / 3) X^{-2}
$$

and for small $X$ this has the same variation in $X$ as (52). Furthermore, if we use $x_{0}^{Z}$ in equation (51) of our analysis we find that at this distance the theoretical surface wave amplitude dominates the space wave amplitude by a factor of $7: 1$, which certainly implies that we can neglect the power in the space wave at this point, hence, the theory confirms the empirical rule.

5. Conclusions. In this report we have investigated a mathematical model of a class of surface wave antennas. A feature of the analysis has been to show that various items of physical interest, such as gain and radiation resistance, can be displayed as elementary mathematical expressions. This has permitted a theoretical interpretation of various facets of operation which while well known from practice, have not been theoretically explained. The results include an estimate of the gain obtainable for a given reactance and antenna length, a demonstration that the surface wave antenna is a non-resonant device since less than $1 \mathrm{db}$ gain can be lost to resonant effects, and a calculation of the purity of the surface wave field as it emerges from the source field. The mathematical inquiry has served to validate a variety of empirical relationships, and illuminate the operating mechanisms of surface wave antennas.

6. Acknowledgment. It is a pleasure for the author to acknowledge the many stimulating discussions with Mr. F. J. Zucker of the Air Force Cambridge Research Laboratories.

\section{Appendix A}

The function $C_{l}\left(r_{l}, \theta_{l} ; X\right)$

From equation (31) of [2] in our notation, $C_{l}$ is given as

$$
C_{l}\left(r_{l}, \theta_{l} ; X\right)=\frac{X \sigma^{+}(k M)}{\left(\cos \theta_{l}-M\right) \sigma^{+}\left(k \cos \theta_{l}\right)} \cdot \frac{\exp }{\left(2 \pi k r_{l}\right)^{1 / 2}} \frac{\left[i\left(k r_{l}-\pi / 4\right)\right]}{(2)}
$$


which has its phase center, $r_{l}=0$, at the discontinuity in reactance $x=l, y=0$; and $\theta_{l}$ is measured counterclockwise from the positive $x$-axis. The factors $\sigma^{+}(k M)$ and $\sigma^{+}\left(k \cos \theta_{l}\right)$ are given explicitly, if approximately, in Appendix B.

\section{Appendix B}

The reflection coefficient $R(X)=\rho e^{i \phi}$ which is used in the text is defined in terms of the decomposition factor $\sigma^{+}(\nu)$ according to the relation

$$
R(X)=\rho e^{i \phi}=X^{2}\left[\sigma^{+}(k M)\right]^{2} /\left[2\left(1+X^{2}\right)\right]
$$

In turn, $\sigma^{+}(\nu)$ satisfies the functional equation

$$
\sigma^{+}(\nu) \sigma^{+}(-\nu)=1-k X i\left(k^{2}-\nu^{2}\right)^{-1 / 2}
$$

where $\sigma^{+}(\nu)$ is analytic in the upper $\nu$-plane $\operatorname{Im} \nu>0$ including the real $\nu$-axis for $\operatorname{Re} \nu>-k$, and $O(1)$ as $|\nu| \rightarrow \infty$. Assume that

$$
\sigma^{+}(\nu)=\sigma^{+}(\nu, k X)
$$

is also analytic as a function of the parameter $k X$, and expand $\sigma^{+}(\nu, k X)$ about $k X=0$.

$$
\sigma^{+}(\nu, k X)=\sigma^{+}(\nu, 0)+k X\left[\sigma^{+}(\nu, 0)\right]^{\prime}+O\left[(k X)^{2}\right],
$$

where the prime denotes differentiation with respect to $k X$. If we set $k X=0$ in (B4), we obtain

$$
\sigma^{+}(\nu, 0) \sigma^{+}(-\nu, 0)=1 \text { implies } \sigma^{+}(\nu, 0)=1
$$

or the leading term in the expansion is unity. To obtain the perturbation factor, we differentiate the defining relationship (B2) with respect to $k X$ and set $k X$ equal to zero

$$
\left[\sigma^{+}(\nu, 0)\right]^{\prime}+\left[\sigma^{+}(-\nu, 0)\right]^{\prime}=-i\left(k^{2}-\nu^{2}\right)^{-1 / 2} \text {. }
$$

Using Cauchy's integral formula, we can decompose $\left(k^{2}-\nu^{2}\right)^{-1 / 2}$ as

$$
\left(k^{2}-\nu^{2}\right)^{-1 / 2}=\left[f^{+}(\nu)+f^{+}(-\nu)\right] / \pi,
$$

where

$$
f^{+}(\nu)=\left(k^{2}-\nu^{2}\right)^{-1 / 2} \ln \left\{\left[i \nu+\left(k^{2}-\nu^{2}\right)^{1 / 2}\right] / i k\right\}
$$

It is a simple matter to show that $f^{+}(\nu)$ is regular in the upper $\nu$-plane $\operatorname{Im} \nu>0$, and a region which includes the real axis for $\operatorname{Re} \nu>-k$. It then follows that

$$
\left[\sigma^{+}(\nu, 0)\right]^{\prime}+\left[\sigma^{+}(-\nu, 0)\right]^{\prime}=-\left[f^{+}(\nu)+f^{+}(-\nu)\right] / \pi,
$$

and by comparing common regions of analyticity on either side of (B9) we conclude that

$$
\left[\sigma^{+}(\nu, 0)\right]^{\prime}=-i f^{+}(\nu) / \pi .
$$

Thus, if we substitute (B5) and (B10) into (B4) we have

$$
\sigma^{+}(\nu, k X)=\sigma_{A}(\nu, k X)+O\left[(k X)^{2}\right],
$$

where the approximate Wiener-Hopf factor $\sigma_{A}^{+}(\nu, k X)$ is

$$
\sigma_{A}^{+}(\nu, k X)=1+(k X / \pi)\left(k^{2}-\nu^{2}\right)^{-1 / 2} \ln \left\{\left[i \nu+\left(k^{2}-\nu^{2}\right)^{1 / 2}\right] / i k\right\}
$$

provided that the proper branches of the radical and logarithm are chosen. 
Verfication. One can readily check that $\sigma_{A}^{+}(\nu, k X)$ shares the same regions of regularity as $\sigma^{+}(\nu, k X)$, and has a similar growth as $|\nu| \rightarrow \infty$. A direct multiplication of the factors

$$
\left[\sigma_{A}^{+}(\nu, k X)\right]\left[\sigma_{A}^{+}(-\nu, k X)\right]=1-k X i\left(k^{2}-\nu^{2}\right)^{-1 / 2}+O\left[\left(k X^{2}\right)\right]
$$

checks the accuracy of the approximation; furthermore,

$$
\sigma_{A}^{+}(0, k X)=\left[1+(X / 2)^{2}\right] \exp \left[-i \tan ^{-1}(X / 2)\right],
$$

which compares well to the known value

$$
\sigma^{+}(0, k X)=\left(1+X^{2}\right)^{1 / 2} \exp \left[-(i / 2) \tan ^{-1} X\right] .
$$

Note: This approximate decomposition depends in large part upon the fact that the coefficient $i\left(k^{2}-\nu^{2}\right)^{-1 / 2}$ of the perturbation factor $k X$ decays more rapidly than the leading term (unity) for $|\nu| \rightarrow \infty$. If the situation were otherwise, then it can be shown [12] that $\sigma^{+}(\nu, k X)$ is not an analytic function of $k X$ about $k X=0$. and the approximation schemes for such cases are a good deal more complex.

\section{Appendix C}

The integral

$$
I=k X \int_{-\infty}^{0} e^{k X \zeta} H_{0}^{(1)}\left(k\left[x^{2}+\zeta^{2}\right]^{1 / 2}\right) d \zeta
$$

can be thought of as a distribution of sources along the negative $\zeta$-axis with a weight factor $e^{k X \zeta}$. Uniformly, for all $\zeta$, and $x$ greater than unity we can argue that

$$
H_{0}^{(1)}(k r) \approx(x / r)^{1 / 2} e^{i k(r-x)} H_{0}^{(1)}(k x)
$$

where $r=\left(x^{2}+\zeta^{2}\right)^{1 / 2}$, or more explicitly

$$
H_{0}^{(1)}(k r) \approx x^{1 / 2} e^{-i k x} H_{0}^{(1)}(k x) \frac{\exp \left[i_{n}\left(x^{2}+\zeta^{2}\right)^{1 / 2}\right]}{\left(x^{2}+\zeta^{2}\right)^{1 / 4}}
$$

so that $I$ becomes

$$
I \approx k X x^{1 / 2} e^{-i k x} H_{0}^{(1)}(k x) J
$$

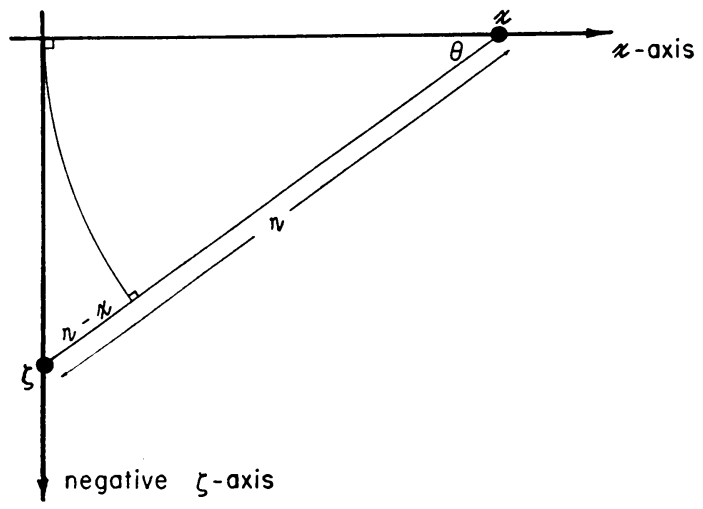

Fra. 5. 
where

$$
J=\int_{-\infty}^{0} \frac{\exp \left[k X \zeta+i k\left(x^{2}+\zeta^{2}\right)^{1 / 2}\right] d \zeta}{\left(x^{2}+\zeta^{2}\right)^{1 / 4}}
$$

Along the contour $S$ which is defined as the locus of points in the complex $\zeta$-plane for which

$$
\mathfrak{g m}\left[k X \zeta+i k\left(x^{2}+\zeta^{2}\right)^{1 / 2}\right]=\text { constant }=k x
$$

the integrand of $J$ decays exponentially. We can deform our path of integration from the negative real axis to that portion of $S$ which lies in the third quadrant because the only singularities $\zeta= \pm i x$ of the integrand lie on the imaginary axes, and the integrand decays exponentially within the shaded region. Hence

$$
J=\int_{S} e^{f(\zeta)} g(\zeta) d \zeta,
$$

where

$$
\begin{aligned}
& f(\zeta)=k X \zeta+i k\left(x^{2}+\zeta^{2}\right)^{1 / 2} \\
& g(\zeta)=\frac{1}{\left(x^{2}+\zeta^{2}\right)^{1 / 4}}
\end{aligned}
$$

and for reference

$$
\begin{aligned}
f^{\prime}(\zeta) & =k X+i k \zeta\left(x^{2}+\zeta^{2}\right)^{-1 / 2} \\
f^{\prime \prime}(\zeta) & =i k\left(x^{2}+\zeta^{2}\right)^{-1 / 2}-i k \zeta\left(x^{2}+\zeta^{2}\right)^{-3 / 2}
\end{aligned}
$$

Let $s=f(\zeta)$ so that

$$
J=\int_{-\infty+i k x}^{0+i k x} e^{s} \frac{g(\zeta)}{f^{\prime}(\zeta)} d s
$$

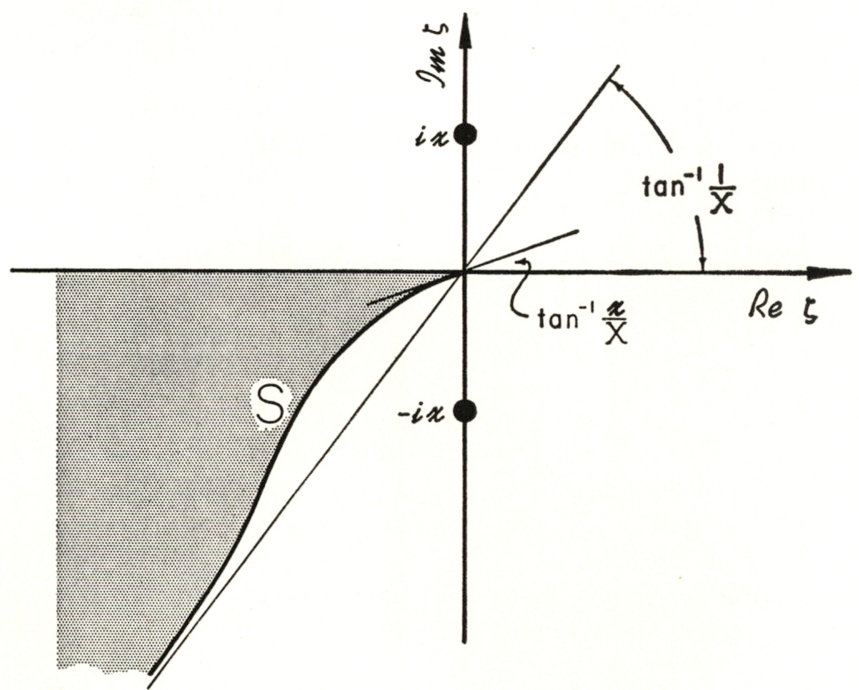

FIg. 6. The contour $S$ in the third quadrant which is the locus of points in the $\zeta$-plane for which $\operatorname{Im}\left[k X \zeta+i \sqrt{x^{2}+\zeta^{2}}\right]=k$. In the illustration we have assumed that $x<X$. 
and a suitable approximation to $J$ is obtained by expanding the algebraic portion of the integrand as a power series in $s$ about $s=0$ and integrating term by term. Note that it is convenient to retain $g$ and $f^{\prime}$ as functions of $\zeta$. The two leading terms of such an expansion would be

$$
\begin{aligned}
J \approx\left[\frac{g}{f^{\prime}}\right]_{\zeta=0} \times \int_{-\infty+i k x}^{0+i k x} e^{s} d s & +\left[\frac{d \zeta}{d s} \frac{d}{d \zeta}\left(\frac{g}{f^{\prime}}\right)\right]_{\zeta=0} \times \int_{-\infty+i k x}^{0+i k x} s e^{s} d s \\
& =\left[\frac{g}{f^{\prime}}\right]_{\zeta=0} \times e^{i k x}-\left[\frac{1}{f^{\prime}}\left(\frac{g^{\prime}}{f^{\prime}}-\frac{g f^{\prime \prime}}{\left(f^{\prime}\right)^{2}}\right)\right]_{\zeta=0} \times e^{i k x} .
\end{aligned}
$$

Since

$$
g(0)=\frac{1}{x^{1 / 2}}, \quad g^{\prime}(0)=0, \quad f^{\prime}(0)=k X, \quad f^{\prime \prime}(0)=\frac{2 i k}{x}
$$

we obtain

$$
J \approx\left[\frac{1}{k X x^{1 / 2}}+\frac{1}{(k X)^{3}} \frac{i k}{x^{3 / 2}}\right] e^{i k x}
$$

and consequently the result

$$
I \approx H_{0}^{(1)}(k x)\left[1+\frac{i}{X^{2} k x}\right]
$$

\section{REFERENCES}

1. S. N. Karp and F. C. Karal, Vertex excited waves on both faces of a right-angled wedge, Comm. Pure Appl. Math. 12, 435-455 (1959)

2. A. F. Kay, Scattering of a surface wave by a discontinuity in the normal reactance with applications to antenna problems, Sci. Rep. No. 7, Technical Research Group, Sommerville, Mass., 1957. Portions of this report were also published in IRE Trans. on Antennas and Propagation, AP-7, 22-31, (1959)

3. B. Friedman and W. E. Williams, Excitation of surface waves, Proc. Inst. Elect. Engrs., 105C, $252-258$ (1958)

4. J. Kane, The efficiency of launching surface waves on a reactive half plane by an arbitrary antenna, IRE Trans. on Antennas and Propagation, AP-8, 500-507 (1960)

5. G. D. Muluzhinets, The excitation, reflection and emission of a surface wave from a wedge with given face impedances, Soviet Physics, Dokl. 3, 752-755 (1958)

6. A. L. Cullen, The excitation of plane surface waves, Proc. Inst. Elect. Engrs. 101, 225-234 (1954)

7. D. B. Brick, The radiation of a Hertzian dipole over a coated conductor, Proc. Inst. Elect. Engrs. 102C, 392-395 (1954)

8. R. E. Plummer, Surface-wave beacon antennas, IRE Trans. on Antennas and Propagation, AP-6, 105-114 (1958)

9. F. J. Zucker, Surface and leaky-wave antennas, Chap. 16, Antenna Engineering Handbook, (ed. Jasik), McGraw-Hill (1961)

10. M. Newstein and J. Lurye, The field of a magnetic line-source in the presence of a layer of complex refractive index, Technical Research Group, Sommerville, Mass., Sci. Rep. No. 1 (1956)

11. S. N. Karp (Private communication)

12. H. C. Kranzer and J. Radlow, Asymptotic factorization for perturbed Wiener-Hopf problems, Adelphi College, Garden City, N. Y., AGM Report No. 101, (1961) 